\title{
Aristotele e Diogene il Cinico
}

DOI: $10.14746 / p e a .2020 .1 .3$

\section{ALDO BR ANCACCI / Università di Roma Tor Vergata/}

Aristotele conosce Diogene, e a lui si riferisce nella Retorica, dove lo cita chiamandolo non con il suo nome proprio, ma unicamente con il suo appellativo di «Cane»: segno che, all'epoca della redazione del terzo libro di quest'opera, Diogene era perfettamente noto ad Atene, e proprio per il suo costume anticonvenzionale di kú $\omega v$. Come ha notato Giannantoni, «ciò è del resto del tutto plausibile in una città non grande come l'Atene del IV sec. a.C. in cui, come prova anche il teatro di Menandro, tutti dovevano conoscersi e facile era la canzonatura reciproca, soprattutto mediante paragoni con gli animali» (Giannantoni 1990: 491). In quale periodo, più precisamente, fissare il riferimento di Aristotele? Fino ad anni recenti è prevalsa l'opinione che la Retorica appartenesse tutta intera alla fase più tarda dell'attività di Aristotele, vale a dire al secondo periodo ateniese. Viceversa, a partire dagli studi di Düring, si è fatta strada la convinzione, oggi largamente accettata dagli studiosi, che i primi due libri della Retorica siano stati composti verso la fine del periodo 360-355 a.C., anche se ciò non esclude la presenza, nell'opera, di parti inserite in anni posteriori, e che il terzo libro della Retorica, che pure costituisce una trattazione 
in certo modo a parte, sia concettualmente e cronologicamente vicino ai primi due. In particolare Düring ritiene che il terzo libro della Retorica sia stato scritto fra il 355 e il 347 a.C. ${ }^{1}$ Entrambe le ipotesi cronologiche, quella tradizionale e quella più recente, si adattano in ogni caso senza difficoltà alla cronologia di Diogene, la cui morte è da porsi attorno al 323 a.C., e che sembra essere giunto in Atene, proveniendo da Sinope, prima del 362 a.C. ${ }^{2}$. Si può quindi concludere che la menzione di Diogene si riferisce al periodo della maturità del filosofo cinico, e non agli anni più tardi o finali della sua vita.

La citazione è compresa nel capitolo 10 del libro III, ove Aristotele, richiamata la classificazione delle metafore in quattro tipi esposta nel capitolo 21 della Poetica, si sofferma sulle metafore $\kappa a \tau$ 'ả $v a \lambda o \gamma$ íav, cioè sulle metafore proporzionali, e cita in successione Pericle, Leptine, Cefisodoro, Ificrate, Pitolao, di nuovo Pericle, Merocle, Anassandride, Polieutte, Cefisodoto, per arrivare infine a Diogene. Anche limitandosi a questi nomi dopo la menzione di Diogene le citazioni continuano ancora - si può notare come la lista aduni espressioni tratte da discorsi pubblici, da intendersi in senso lato, contenendo frasi di oratori, prevalentemente, ma anche di uomini politici, di un poeta comico e di un filosofo. Il passo che riguarda il Cane è il seguente:

Dei quattro tipo di metafora, ottengono maggior successo quelle per analogia, come Pericle disse che la giovinezza morta nella guerra era scomparsa nella città come se si fosse sottratta la primavera all'anno. (...) E Cefisodoto chiamava le triremi «mulini multicolori», e il Cane definiva le taverne «le mense comuni dell'Attica ${ }^{3}$.

È congettura senza fondamento di Goulet-Cazé che con l'appellativo ó Kúwv Aristotele si riferisca qui non a Diogene, come tutti gli studiosi convengono, bensì ad Antistene ${ }^{4}$. La studiosa non reca, peraltro, nessuna prova determinata a sostegno di tale congettura, ma solo una serie di rilievi, che non tengono conto di numerosi dati obiettivi contrari alla sua ipotesi, e che sono stati recentemente sottoposti a una minuta analisi e confutatis. C’è stato un tempo, nella storiografia filosofica, in cui Antistene era senz'altro assimilato al Cinismo a lui posteriore: erano i tempi, ormai molto lontani, della storiografia

${ }^{1}$ Si veda su ciò Düring (1966: 50, 121-124). Cfr. inoltre Gastaldi (2014: 34, n. 9). Sull'importanza della testimonianza aristotelica ai fini della determinazione della cronologia di Diogene si sofferma anche Döring, (1995: 134): «Diogenes muss seine auffällige Lebensweise zu dieser Zeit in Athen also schon so lange praktiziert haben, dass er jedermann unter dem Namen „der Hund“ bekannt war».

2 Per la cronologia di Diogene rinvio all'accurato riesame che di tutte le testimonianze e di tutti i dati a nostra disposizione ha svolto Giannantoni (1990: 421-433), il quale giunge alla conclusione che Diogene sia nato nel periodo 412/403 e che sia morto negli anni 324/321.

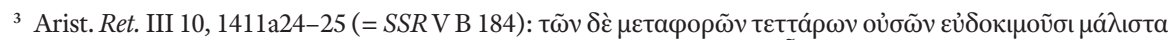

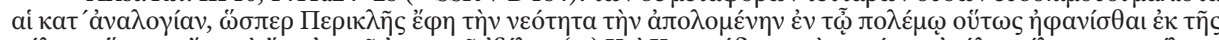

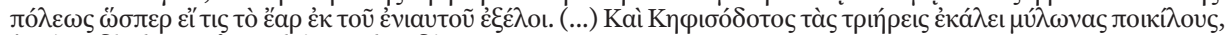

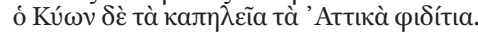

${ }^{4}$ Cfr. Goulet Cazé (1996: 414-415).

${ }^{5}$ Cfr. Zaccaria (2017), il quale ha confermato con una analisi particolare la corretta identificazione del Kú $\omega v$ menzionato da Aristotele con Diogene il Cinico. 
ottocentesca e primonovecentesca. Questa prospettiva è da tempo tramontata: tutto il lavoro della storiografia posteriore è consistito nel ristabilire una più esatta prospettiva storica e teorica, e nello sfaldare quella facile e infondata assimilazione. Indubbiamente tra Antistene e il Cinismo esistono delle connessioni, ma esse non riguardano il кuvıкò ßíos, che è una creazione di Diogene, la quale, anzi, sarà completata solo dal cinismo della generazione a lui successiva, e sottoposto a ulteriori trasformazioni nella fase più tarda del cinismo. Va inoltre ricordato che è Diogene, non Antistene, ad essere e a rappresentare "il Cane" in tutta la tradizione antica, letteraria e biografico-dossografica: da Diogene Laerzio alle Lettere pseudodiogeniche, da Dione Crisostomo a Temistio, da Epitteto a Luciano. Si aggiunga, e questo è determinante, che, nei numerosi passi della sua opera nei quali si riferisce ad Antistene ${ }^{6}$, Aristotele lo chiama sempre e senza ecce-

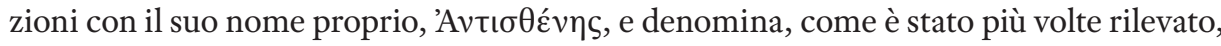

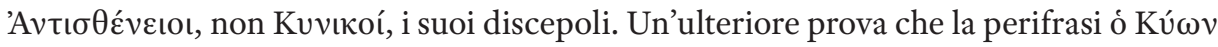
indica non Antistene, ma un altro filosofo, diverso da lui, è data dal fatto che Antistene stesso è citato da Aristotele proprio nella Retorica, nel terzo libro, con il suo nome proprio. Aristotele riporta un gustoso paragone usato dal Socratico in uno dei suoi scritti, e usa

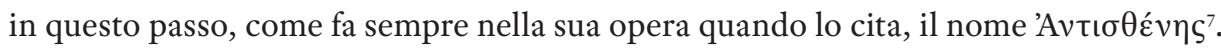
È inverosimile che nel primo libro della medesima opera egli voglia alludere ad Antistene, chiamandolo però questa volta (e unicamente questa volta), improvvisamente, e senza alcuna altra specificazione, ó Kúwv.

L'ironia del motto di Diogene, il quale assimila le taverne attiche (

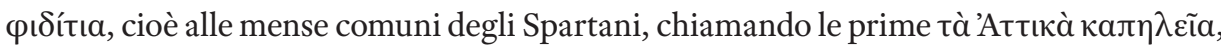
consiste, come ha chiarito Gastaldi, «nel mettere sullo stesso piano le taverne ateniesi, che sono sede di comportamenti sregolati, dovuti all'uso incontrollato di vino e di cibo, e le mense collettive spartane, austere e rigidamente controllate ${ }^{8}$. Chiamare le sregolate taverne attiche con il nome che spetta alle austere mense collettive lacedemoni significa, nello stesso tempo, coniare una metafora e investirla di un pungente significato ironico, anzi sarcastico. Il tema dello «stare nelle taverne» è attestato anche altrove in Diogene e nella tradizione cinica, con utilizzazioni e con sfumature di significato diverse, volte a rilevare la capacità del saggio cinico di recarsi in ogni luogo mantenendo inalterata la sua sovranità sulle situazioni esterne, la sua libertà e il suo autodominio9 . Lo stesso

${ }^{6}$ Sul modo in cui Antistene è presentato in queste testimonianze si vedano Sayre (1938: 52), e Aubenque (1963: 146, n. 2); sul contenuto teorico di queste testimonianze, Brancacci (2005: 195-223).

7 Arist. Ret. III 3, 1407a9-12 (= SSR V A 51). Su questa testimonianza, cfr. Prince (2015: 181-182).

8 Cfr. Gastaldi (2014: 581).

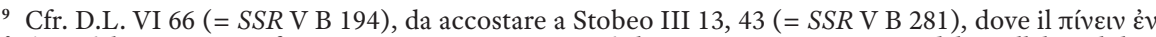
$\kappa a \pi \eta \lambda \varepsilon \varepsilon^{\prime} \omega$ può ben essere confacente a Diogene, se - questo è il sottinteso, come si ricava dal parallelo stabilito con il fatto di farsi tagliare i capelli dal barbiere - tale azione è compiuta in modo naturale e in termini coerenti con il semplice modo di vita cinico; e inoltre Eliano ( $V H 9,19=S S R$ V B 503), che ci restituisce un aneddoto

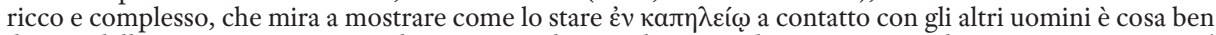
diversa dallo stare a contatto con gli uomini per lusingarli o per ridursi a esserne schiavi, come avviene a oi

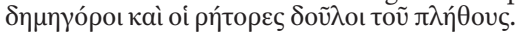


può dirsi della lode dei costumi degli Spartani, insieme con il biasimo del modo di vita rilassato degli Ateniesi, o semplicemente della massa degli uomini, che è anch'esso tema tipicamente diogenico e cinico $^{10}$.

La citazione di Aristotele è notevole per almeno due ragioni. Per un verso, essa è la testimonianza più antica in nostro possesso su Diogene, nonché sulla sua aderenza ad almeno alcuni aspetti del кuvıкòs ßíos, che Diogene rappresenta emblematicamente, a tal punto da poter essere citato semplicemente come «il Cane»; e, inoltre, sulla sua notorietà ad Atene attorno alla metà del quarto decennio del IV secolo. Per un altro verso, esso dimostra, anche, che Aristotele è in grado di citare uno scritto di Diogene, peraltro con apprezzamento. La testimonianza di Aristotele acquista così un valore emblematico per chi persegua il tentativo di ricostruire la figura di Diogene sulla base di documenti fededegni e autorevoli, che è necessario attentamente selezionare tra la massa di apoftegmi e rappresentazioni di non chiarita attendibilità, onde sfuggire a quelle rappresentazioni puramente letterarie e pittoresche della figura di Diogene che ancora oggi sono dure a morire negli studi. Tale testimonianza ci presenta infatti un Diogene che può essere denominato semplicemente «il Cane», il che mostra come egli sia un filosofo dedito

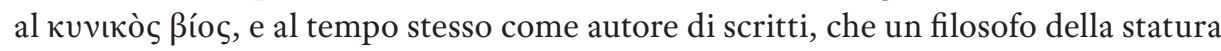
di Aristotele reputa degni di essere citati. Diogene è presentato cioè anche come un autore letterariamente orientato, come, del resto, attestano anche altri documenti particolarmente importanti della tradizione a lui relativa.

Qui è sufficiente ricordarne due. In primo luogo l'estratto di Eubulo, conservato da Diogene Laerzio, nel quale Diogene è rappresentato nelle vesti di un maestro cinico,

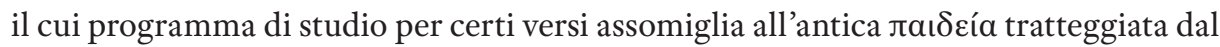
Discorso Giusto nelle Nuvole di Aristofane: egli si prende cura dei giovinetti a lui affidati impartendo loro una educazione naturale e severa, ma nello stesso tempo cura la formazione culturale dei suoi allievi facendo loro studiare e imparare a memoria brani di poeti e prosatori, e, inoltre, opere da lui stesso composte ${ }^{11}$. A ciò corrisponde la testimonianza, che ovviamente è ancora più importante, offerta dai cataloghi degli scritti di Diogene conservati da Diogene Laerzio. Il primo catalogo, che è quello più autorevole, benché anonimo, comprende tredici Dialoghi, sette Tragedie, e le Epistole; il secondo, di minore valore perché riflette le preoccupazioni stoiche di negare l'autenticità di opere fondamentali di Diogene quali la Repubblica e le Tragedie, giudicate irriverenti per il loro contenuto, mantiene comunque menzione di vari Dialoghi. Entrambi, in aggiunta a quanto risulta da altre testimonianze antiche, e unitamente a quanto hanno potuto stabilire numerosi

${ }^{10}$ Cfr. ad esempio D.L. VI 27 (= SSR V B 280) «Essendogli stato chiesto in quale luogo dell'Ellade avesse visto uomini buoni, rispose: "Uomini buoni in nessun luogo, ragazzi buoni a Sparta"»; D.L. VI 59 (= SSR V B 282). Per i giudizi sugli Ateniesi, Spartani, Megaresi etc. si vedano tutti i testi raccolti in SSR V B 280-288.

11 Cfr. D.L. VI 30-31 (= SSR V B 70), e su di essa si veda Höistad (1948: 116-126 e 176-179); cfr. inoltre Barns (1951: 7-8); Donzelli (1960: 248-253); Giannantoni (1990: 485-489). 
studi moderni dedicati a tali scritti ${ }^{12}$, confermano, come è stato più volte osservato, che Diogene è presentato dalle fonti antiche come filosofo in grado di citare e parodiare versi di Omero e dei tragici, di comporre egli stesso tragedie, aventi valore dottrinale-didascalico, nonché di padroneggiare le risorse della retorica, piegata a un uso personale e, ancora una volta, volta a obiettivo filosofico.

Ma perché Aristotele chiama Diogene ó kú $\omega v$ ? Un primo elemento di risposta, che sicuramente non è sufficiente a chiarire tutte le ragioni dell'epiteto guadagnatosi dal filosofo cinico, ma che certo ne illumina almeno un aspetto, giunge dalla stessa metafora di Diogene citata da Aristotele. Essa indica la mordacità di Diogene, la sua capacità di riprendere gli Ateniesi per i loro costumi e il loro modo di vita, insomma la caratteristica funzione del filosofo cinico di fustigare e ammaestrare gli uomini, in aderenza a quella rappresentazione del cane come 'animale filosofico' che, in definitiva, risale ad Antistene e al suo scritto Пвpì toũ $\kappa u v o ́ \varsigma^{13}$. Per essa, il cane è l'animale capace di distinguere ciò

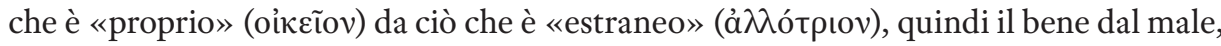
e conseguentemente l'amico (l'uomo buono) dal nemico (l'uomo malvagio). È molto probabile che Diogene abbia ripreso e sviluppato questa caratterizzazione del cane come

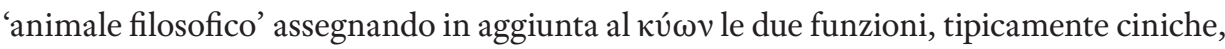

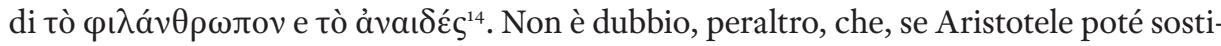
tuire il nome di Diogene semplicemente con quello de «il Cane», ciò fu possibile solo perché Diogene rappresentava emblematicamente i modi del кuvıкò ßíos, che in effetti nasce con lui, come, compatta, la tradizione antica attesta. Tra le tante testimonianze disponibili, è utile ricordare anche una testimonianza indiretta, particolarmente preziosa, di Plauto, dalla quale si può desumere la descrizione dell'abbigliamento del filosofo cinico ai tempi del cinismo più antico:
Cynicum esse egentem oportet parasitum probe:
ampullam, strigilem, scaphium, soccos, pallium, marsuppium habeat, inibi paullum praesidi, qui familiarem suam vitam oblectet modo ${ }^{15}$.

12 Sull'attività letteraria di Diogene, e sui due cataloghi laerziani, si veda la ricca nota Gli Scritti di Diogene di Giannantoni (1990: 461-484), cui rinvio anche per ulteriori indicazioni bibliografiche. In particolare sulla Repubblica e sulle Tragedie sono da ricordare Bartalucci (1970-1971); Schofield (1991: 10-13 e 141-145); Dorandi (1993); Moles (1995: 129-143); Husson (2011); López Cruces (2003; 2010).

${ }^{13}$ Per questo scritto, registrato nel nono tomo del Catalogo degli scritti di Antistene, cfr. D.L. VI 18. Su di esso si vedano le note di Decleva Caizzi (1966: 85), e di Giannantoni (1990: 336-337). Weber (1887: 87), riteneva, giustamente, che in quest'opera il cane (da identificarsi con Argo, il cane di Odisseo, come mostra la posizione di questo scritto nel nono tomo del Catalogo, comprendente, insieme con l'ottavo, le opere di esegesi

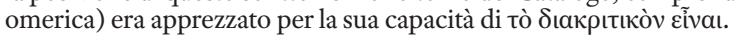

${ }^{14}$ Come risulta, ad es., da Eust. Ad Od. VII 315, su cui cfr. Decleva Caizzi (1993: 315).

15 Pl. Per. $125-128$ (= SSR V B 162). 
Se, come Leo ha argomentato, e come anch'io sono propenso a credere, l'originale della commedia plautina risale all'età di Demostene, ne segue che in essa non si parlava dei cinici in generale, i quali erano ancora di là da venire, ma proprio di Diogene, o almeno dell'immagine che egli del cinico aveva lasciato ${ }^{16}$. Anche se si supponesse, come recentemente è stato sostenuto, che essa rinvii piuttosto a Cratete di Tebe e ai cinici della sua epoca (Izzo 2014), in entrambi i casi resteremmo nell'ambito del cinismo delle origini. E in effetti la cosa importante da notare, qui, è che i tratti caratteristici dell'abbigliamento del cinico coincidono solo in parte con quelli che saranno propri dei cinici d'età imperiale e che nella storia degli studi sono stati proiettati retrospettivamente sul cinismo delle origini, o, ancor peggio, considerati tratti astorici e perenni. In effetti, nel passo citato il cinico indossa un pallium (= $\tau \rho \hat{\beta} \beta \omega v$ ), senza alcuna sottoveste, e, particolare degno di nota, porta le scarpe; ha un marsuppium, che non è la bisaccia (= di piccolo borsello; manca inoltre in Plauto la menzione del bastone e della bisaccia, che appartengono al costume del girovago, e divennero solo dopo Diogene il ritratto consueto del cinico, ritratto che fu poi proiettato a ritroso su Diogene stesso. La mancanza della $\pi n ́ \rho a$, e la presenza invece del marsuppium, è un'altra prova a favore del riferimento di questo abbigliamento a Diogene, piuttosto che a Cratete. Questi fu autore addirittura di un poema elegiaco intitolato Пń $\rho \eta^{17}$, eletta a simbolo della sua professione di cinismo, e non è verosimile che, se quel ritratto si riferisse a lui o ai tempi suoi, proprio la «bisaccia» mancasse. È invece del tutto verosimile che il marsuppium costituisse il precedente di quella ли́рa che a partire da Cratete diventerà un elemento caratteristico dell'abbigliamento cinico. Particolarmente significativa è poi la menzione della ampulla e dello strigilis, che sono elementi tipicamente attici, senza i quali non è pensabile la vita di un Ateniese, i quali ci riportano precisamente a Diogene. Ciò ci fa comprendere che è da questa che Diogene ha preso le mosse, accompagnandola con un procedimento di progressiva sottrazione, sottrazione della quale è rimasta memoria anche negli aneddoti più tardi, alcuni dei quali celebri, aventi però per lo più valore solo simbolico, e ben poca consistenza storica ${ }^{18}$.

Questo processo di progressiva sottrazione è altro tema che trova la sua origine in Antistene, presso il quale è presente, e ampiamente e autorevolmente attestato, un tema

16 Cfr. Leo (1906 [1960]). Leo prende le mosse da U. von Wilamowitz-Moellendorff (1893: 16 [1935: 190]). Ha ripreso in modo approfondito la questione, confermando con molte osservazioni la tesi di Leo e Wilamowitz, Giannantoni (1990: 499-503). Sulla commedia plautina cfr. anche Pasetti (2011).

${ }_{17}$ Cfr. D.L. VI 85 (= SSR V H 70). Su di esso, e sul pensiero di Cratete, si vedano Höistad (1948: 128-131), Criscuolo (1970); Giannantoni (1990: 561-579), al quale rinvio anche per ulteriori indicazioni bibliografiche.

18 Il movimento di progressiva sottrazione è ben testimoniato, ad esempio, da testi quali D.L. VI 23 (= SSR V B 174), i quali documentano la genesi del modo di vita cinico di Diogene, e i suoi momenti, non necessariamente tutti compresenti fin dall'inizio, ma acquisiti col tempo: «In un primo tempo si appoggiava al bastone solo quando era ammalato, ma successivamente lo portava sempre, non tuttavia in città, ma quando camminava lungo la strada, insieme con la bisaccia: così riferiscono Olimpiodoro, colui che governò gli Ateniesi, e il retore Polieucto e Lisania, figlio di Escrione». Il passo successivo è anch'esso interessante, soprattutto nella sua prima parte, la quale mostra due cose: da un lato, ancora una volta, la genesi, per sottrazione di comodità e "spostamento" di scelte o obiettivi, del modo di vita cinico di Diogene: dall'altro il fatto che Diogene, prima di procedere a tale sottrazione e spostamento di interessi, non disdegnava di provvedersi (e quindi possedeva 
molto vicino a quello diogenico: il concetto di riduzione dei bisogni, situato in un oriz-

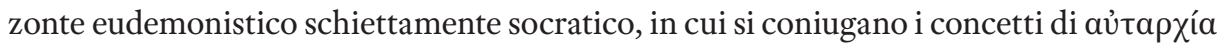
e di aủtá $\rho \kappa \varepsilon ı \alpha^{19}$. Esso è confermato, inoltre, dalla testimonianza su Diogene che, qualche decennio dopo Aristotele, ci rende il suo discepolo Teofrasto. Di particolare rilievo non solo per la sua antichità, ma anche per la sua imparzialità e per il suo interesse scientifico, essa è desunta da un suo scritto, il Megarico, e ci è riportata da Diogene Laerzio:

Teofrasto nel suo Megarico narra che una volta [sc. Diogene] vide un topo correre qua e là, senza mèta. Non cercava un luogo per dormire, né aveva paura delle tenebre, né desiderava alcunché di ciò che viene considerato desiderabile. E così ritrovò il rimedio alle sue difficoltà ${ }^{20}$.

Teofrasto doveva trattare di Diogene il Cinico, come già hanno sostenuto vari studio$\mathrm{si}^{21}$, anche in un'altra sua opera, registrata nel catalogo laerziano (Diogene Laerzio V 43),

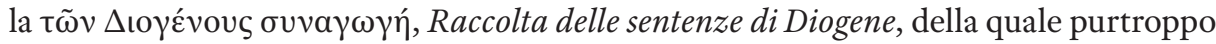
non ci sono pervenuti frammenti. Il Diogene che figura nel titolo dell'opera è anche a mio giudizio il Cinico. L'obiezione, secondo cui sarebbe improbabile che Teofrasto parlasse del Kú $\omega v$ in due opere distinte, è inconsistente: solo la $\Sigma v v a \gamma \omega \gamma \eta ́$ era infatti dedicata a Diogene, mentre il Megarico non era a lui intitolato: non ne trattava quindi ex professo, e, come risulta dal passo citato, doveva, oltretutto, trattare anche di altri personaggi, quali Atenodoro, Polieucto e Lisania.

La testimonianza di Teofrasto conferma, dopo Aristotele, l'interesse dell'ambiente peripatetico per Diogene e il cinismo: che del resto è reciproco, posto che uno dei maggiori esponenti del cinismo antico, Metrocle di Maronea, fu discepolo di Teofrasto

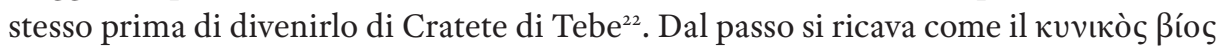
trovi la sua genesi nel principio dell'economia dei bisogni, e nell'osservazione della vita

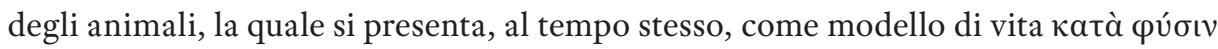
e come esempio di una condotta che realizza una perfetta adeguazione tra mezzi e fini, tra

i mezzi per provvedersi) di una casetta, il che a sua volta richiama la posizione di Antistene nella descrizione del suo modo di vita parco ma sufficiente e non ascetico nella autopresentazione di Antistene stesso nel Simposio senofonteo (si veda la successiva nota 15): «Una volta aveva ordinato a un tale di provvedergli una casetta; poiché quello indugiava, egli si scelse come abitazione una botte che era nel Metròo, come attesta egli stesso nelle Epistole». Le Epistole di cui qui si parla sono, ovviamente, quelle autentiche di Diogene, registrate sia nel primo Catalogo degli scritti riportato da Diogene Laerzio, sia nel secondo Catalogo, di Sozione, riportato anch'esso in D.L. VI 80.

19 X. Smp. 4, 34-44 (= SSR V A 82). Ho studiato questo passo nel mio saggio (Brancacci 1993).

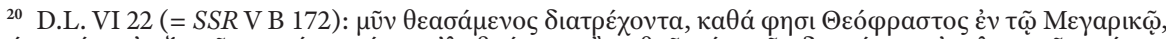

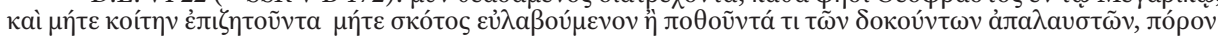

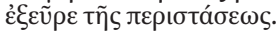

${ }^{21}$ Grote (1888: 508); von Fritz (1926: 36-39); Dudley (1937: 6-7); Gallo (1980: 248-249).

${ }^{22}$ Cfr. D.L. VI 94 (= SSR V L 1). 
bisogni e soddisfazione dei medesimi, mettendo anche su questo punto in luce il fondamentale razionalismo del cinismo di Diogene.

Chiarito che Aristotele conosce Diogene, ed è in grado di citare un suo scritto, possiamo ora procedere all'esame di un passo tratto dalla Politica dello stesso Aristotele. Questo passo è molto importante perché consente di conferire evidenza teorica ad alcuni tasselli fondamentali della concezione politica di Diogene. Esso è compreso in una sezione del primo libro della Politica e si riferisce molto probabilmente, come vedremo, proprio a Diogene; e, se è così, non è da escludersi che Diogene stesso possa averlo conosciuto, e che a sua volta abbia meditato tutti i capitoli iniziali della Politica, ai quali risponderebbe con le tesi e le prescrizioni di segno opposto contenute nella Repubblica.

Prima di esaminarlo, occorre ricordare che nel primo libro della Politica Aristotele aveva dapprima scritto:

chi non è in grado di entrare nella comunità o per la sua autosufficienza non ne sente il bisogno, non è parte della città, e di conseguenza è o bestia o dión ${ }^{23}$.

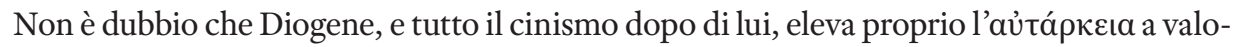
re supremo, e che, sposandola, la assume proprio ponendosi nel ruolo dell'animale ${ }^{24}$. È da ricordare anche che nel passo riportato Aristotele intende dimostrare l'anteriorità della polis rispetto all'individuo: una tesi eminentemente antisocratica, che Diogene non avrebbe potuto condividere, posto che tutta l'azione del cinismo, e prima ancora del socratismo, in sede politica, è stata quella di trasformare la polis e i suoi valori a partire da una riforma in senso etico dell'individuo ${ }^{25}$. Progetto utopistico, forse, ma certamente coerente sia con il senso, costantemente ribadito da Socrate, del suo ufficio, sia con le dichiarazioni esplicitamente formulate da Diogene nella sua Repubblica, e poi con tutta la concreta azione, etica e politica, svolta dai cinici. Più complessa è la situazione teorica su altri punti di questa parte iniziale della Politica. Aristotele ritiene che la polis è costituita dalla riunione di più villaggi, e che la polis perfetta è quella che raggiunge il limite della propria completa autosufficienza, talché, sebbene nata per rendere possibile la vita, essa di fatto esiste per rendere possibile una vita felice: un punto di vista che Diogene avrebbe potuto accogliere, se riferito, però, non alla polis storica, ma a quella da lui ridisegnata nella Repubblica. Ancora più interessante è il seguito dell'argomentazione aristotelica:

Quindi ogni stato esiste per natura, se per natura esistono anche le prime comunità: infatti esso è il loro fine e la natura è il fine: per esempio quel che ogni cosa è quando ha compiuto il

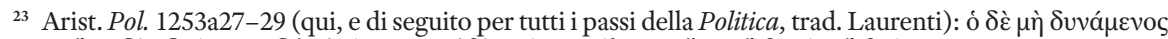

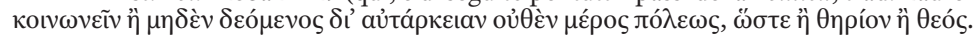

${ }^{24}$ Cfr. Dierauer (1977: 180-193).

${ }^{25}$ Per questo, cfr. Brancacci (2011).
} 
suo sviluppo, noi lo diciamo la sua natura, sia d'un uomo, d'un cavallo, d'una casa. Inoltre, ciò per cui una cosa esiste, il fine, è il meglio e l'autosufficienza è il fine e il meglio ${ }^{26}$.

L'autosufficienza è dunque a due riprese considerata da Aristotele come un fattore positivo: rispetto alla città, in quanto delimita l'organizzazione che essa deve raggiungere e alla quale deve provvedere; e rispetto al concetto di natura, in relazione alla quale di nuovo l'autosufficienza è il fine e il meglio. Essa è invece negativamente considerata quando, soggettivamente vissuta, sia tale da spegnere il desiderio dell'uomo di entrare nella comunità. Ora, il punto di disaccordo più evidente tra Aristotele e Diogene risiede proprio nella determinazione del concetto di natura. Per Aristotele, essa designa lo stato che una cosa o un vivente, sia esso uomo o animale, raggiungono quando hanno compiuto il loro proprio sviluppo, sviluppo che nel caso dell'uomo deve partire dal suo essere un animal politicum, con tutte le implicazioni che questo concetto ha nella Politica; per Diogene, essa risiede in una economia dei bisogni, dei desideri e dei comportamenti tale da realizzare un modo di vita nuovo, appagato, fondato su una critica e anzi sul capovolgimento di tutti i valori ancorati alla sfera del nomos. Il secondo punto di disaccordo tra Aristotele e Diogene è nella definizione della polis come tale che essa è «per natura» ( $\varphi v ́ \sigma \varepsilon ı)$ : per Diogene essa è certamente «per convenzione» ( $\nu$ ó $\omega$ ), e nella convenzione rientra anche la storia, immaginata o reale, che dalla famiglia conduce ai villaggi e dalla pluralità di villaggi alla città, perché, per lui, sulla convenzione sono basati i rapporti familiari e la vita associata degli uomini.

Da questo punto di vista, è interessante notare come la parte finale del secondo capitolo del primo libro della Politica, e i capitoli immediatamente successivi, si intrattengano su una serie di temi e problemi sui quali il cinismo ha fatto sentire la sua voce, ma in una direzione opposta a quella teorizzata qui da Aristotele. Due temi in particolare meritano attenzione: nell'ambito della trattazione relativa all'amministrazione domestica, la definizione del rapporto padronale; nell'ambito della trattazione della crematistica, la celebre distinzione tra i due tipi di crematistica, e infine la trattazione relativa alla genesi della moneta. Nel primo caso, Aristotele, è noto, accetta la legittimità e anche naturalità della distinzione tra padrone e schiavo, e dell'autorità del primo sul secondo, che del resto va parallela alla teorizzazione della sottomissione e inferiorità della donna all'uomo, per cui questi comanda, quella è comandata ${ }^{27}$. Sappiamo invece che il cinismo propugna un atteggiamento paritetico nei confronti della donna (già Antistene aveva affermato: «la stessa è la virtù dell'uomo e della donna ${ }^{28}$ ) e certamente aperto verso gli schiavi

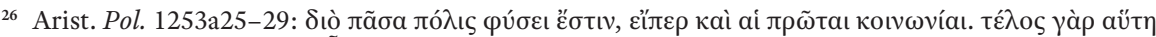

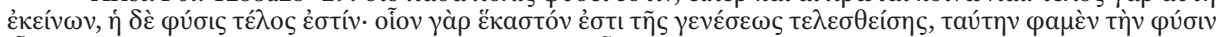

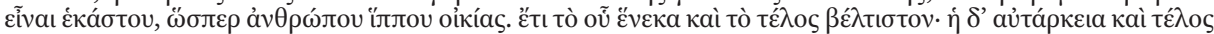
каì $\beta \varepsilon ́ \lambda$ tıбтоv.

${ }^{27}$ Arist. Pol. 1254b13-15: «Così pure nelle relazioni del maschio verso la femmina, l'uno è per natura superiore, l'altra inferiore, l'uno comanda, l'altra è comandata».

${ }_{28}$ Diocle apud D.L. VI 12 (= SSR V A 134). 
in senso proprio, riservando l'appellativo metaforico di schiavi agli uomini liberi, privi però di ragione ${ }^{29}$. Nel secondo caso, la contrapposizione alla ricca e complessa spiegazione aristotelica dell'introduzione e della funzione della moneta è ancora più evidente: nella Repubblica, Diogene propugna l'abolizione della moneta, come, d'altronde, abolisce il matrimonio e ridisegna, improntandoli a una radicale libertà sessuale, i rapporti tra uomo e donna ${ }^{30}$. La celebe kynogamia, rappresentata dalle nozze di Cratete e Ipparchia, un tempo raffinata donna d'alto lignaggio, esemplifica questa concezione, che implica anche una serie di atti simbolico-concreti: l'abbandono delle ricchezze, l'abbandono delle vesti sontuose o abituali, l'abbandono della dimora natale, l'assunzione della vita errante

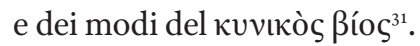

L'ipotesi che Diogene possa avere meditato questi testi di Aristotele, costruendo per opposizione la sua teoria, è resa ancora più probabile dal fatto che in questi stessi passi della Politica Aristotele registra l'esistenza di una posizione teorica, relativa alla moneta, che richiama molto da vicino proprio quella di Diogene. Ecco il passo in questione, al cui esame possiamo finalmente giungere:

Al contrario, taluni ritengono la moneta un non-senso, una semplice convenzione legale, senz'alcun fondamento in natura, perché, cambiato l'accordo tra quelli che se ne servono, non ha più valore alcuno e non è più utile per alcuna delle necessità della vita, e un uomo ricco di denari può spesso mancare del cibo necessario: certo, strana davvero sarebbe tale ricchezza, che, pur se posseduta in abbondanza, lascia morire di fame, come appunto il mito tramanda di quel famoso Mida, il quale, per il voto suggerito dalla sua insaziabilità, trasformava in oro tutto quanto gli si presentava ${ }^{32}$.

Il passo presenta un evidente riferimento alla caratteristica tesi di Diogene relativa alla svalutazione e devalorizzazione della moneta, tesi che ci è attestata da varie fonti. Dalla testimonianza, antica e preziosa, di Filodemo, apprendiamo che nella sua Repubblica Diogene stabilì per legge che la moneta (vó $\mu \sigma \mu \alpha)$ fosse costituita da astragali, evidentemente perché, essendo la moneta fatto puramente convenzionale, non solo essa avrebbe potuto essere costituita da qualunque oggetto o manufatto, ma nulla più di un astragalo avrebbe potuto rappresentarne l'intrinseco valore convenzionale, proprio in accordo con la tesi complessiva ricordata da Aristotele nel passo citato. Ed è da ricordare che

\footnotetext{
${ }^{29}$ Basti rinviare ai testi SSR V B 440-445. Si ricordino inoltre il decimo discorso di Dione Crisostomo, Diogene o sui servi, e i Fuggitivi di Luciano.

${ }^{30}$ Per testi e discussione, su questi temi, rinvio a Husson (2011: 105-145).

${ }^{31}$ Sulla kynogamia cfr. Giannantoni (1990: 565-566). Su Ipparchia si vedano, tra i contributi più recenti, Baroncelli, Lapini (2001); Lapini (2003: 217-230); Paradiso (2014).

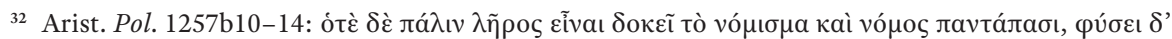

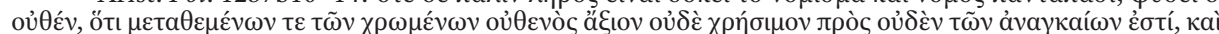

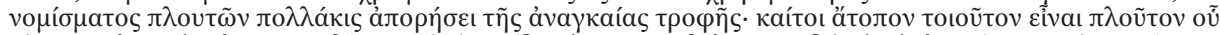

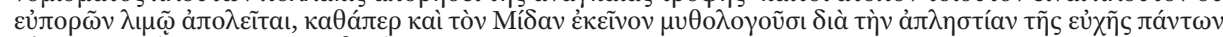

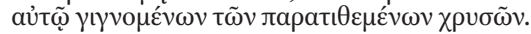


l'attestazione di Filodemo è ripresa alla lettera da Ateneo, il quale ripete anche il preciso e impegnativo verbo usato dall'epicureo, per caratterizzare la prescrizione di Diogene:

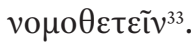

Gli studiosi hanno discusso sul significato di questa tesi, e si sono divisi tra coloro che, come Gomperz, hanno supposto che il Cinico avesse in mente un progetto economico ben preciso e concorrenziale rispetto a quello corrente ${ }^{34}$, e altri che, come Moles, hanno preferito interpretare la tesi diogenica come un mero «joke» ${ }^{35}$. Ora, Moles coglie bene l'elemento ironico, e anche giocoso e scherzoso, che accompagna tutti gli atti e le prescrizioni anticonvenzionali di Diogene, e che in misura variabile ma costante è presente in tutte le sue posizioni e attitudini più estremistiche e presuntamente scandalose: ciò non toglie che esse siano perfettamente serie, come lo è la tesi dell'abolizione della moneta e della sua sostituzione con semplici astragali. Inoltre, va ricordato che lo scherzo, in Diogene, non solo non esclude la serietà, ma anzi la coimplica. Si ricordi che tò

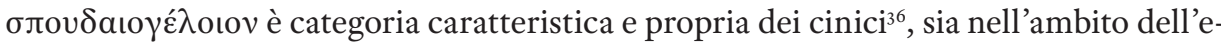
spressione letteraria, sia come indicatore di uno stile di pensiero, che, a sua volta, non fa che riprendere, sviluppare e portare alle estreme conseguenze l'ironia di Socrate. Inoltre, l'espressione usata da Filodemo, e ripresa alla lettera da Ateneo, vo $\mu$ o $\theta \varepsilon \tau \varepsilon \tilde{v}$, non lascia dubbi: essa indica l'atto della prescrizione legale, e, in quanto tale, mostra che la tesi stessa non solo non è affatto un mero scherzo, ma fa parte del progetto politico, provocatorio e anticonvenzionale quanto si vuole, ma certamente razionale e positivamente assunto,

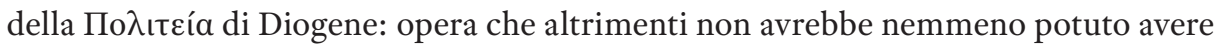
e ricevere questo titolo. Senza contare che tutta l'Antichità ha ritenuto perfettamente serie le tesi contenute nella Repubblica, e proprio per questo ne ha serbato scandalizzata memoria, a tal punto da arrivare, con una certa linea degli Stoici, a negarne l'autenticità, per le ragioni che tutti conoscono e che da tempo sono state chiarite.

Ora, il passo di Aristotele ci restituisce proprio quello che manca negli scarni enunciati di Filodemo e di Ateneo: la ragione per la quale Diogene proponeva, con ragioni del tutto serie, l'abolizione del conio corrente, e la sua sostituzione con semplici astragali. L'argomentazione è chiara e lineare, e la si può agevolmente così ricostruire: la moneta non ha alcun fondamento in natura; essa è puro fatto di vó $\mu$ os: se cambia la convenzione stabilita tra coloro che l'hanno istituita, la moneta, considerata in sé stessa, non ha più alcun valore, e non serve a niente, men che mai alle necessità basilari della vita; l'unica cosa cui la moneta serve è quello di consentire lo scambio: ma allora a questa funzione possono benissimo assolvere gli astragali, oggetti privi di ogni valore intrinseco, che

${ }^{33}$ Cfr. Phld. De Stoicis, XVI 4-11 (= SSR V B 126); Ath. Deipnosophistae, IV 159c (= SSR V B 125).

${ }^{34}$ Gomperz ritiene la proposta di abolire la moneta e di sostituirla con gli astragali come «die Einführung einer Art von Papiergeld» (Gomperz 1903: 132). A questa tesi si oppose già Giannattasio Andria (1980:139, n. 35), senza però fornire argomentazioni precise e determinate, e solo dichiarando scarsamente verosimile la presenza di interessi di natura economica in Diogene.

35 Cfr. Moles, il quale ha ritenuto la proposta diogeniana un mero «joke» (Moles 1995: 137).

${ }^{36}$ Cfr. Dudley (1937: 110-116); Roca Ferrer (1974). 
Diogene sceglie come sostituto del conio di corso legale, che è investito di intrinseco valore pecuniario, proprio perché essi ne sono, visibilmente, del tutto privi. In questo modo gli astragali fungeranno bensì da moneta, ma storneranno gli uomini da quella follia consistente nel conferire valore a monete, ori e ricchezze che o non servono affatto ai bisogni della vita, o la corrompono. Non è dunque il caso di parlare di principi di «economia» in senso stretto, concetto in larga misura anacronistico e scarsamente adeguato a cogliere gli interessi di Diogene, ma neppure è il caso di parlare di un mero «scherzo»: la sua è una tesi di filosofia politica, del tutto seria, che, dal punto di vista storico-filosofico, disegna una linea di continuità tra sofistica e cinismo, ove il secondo radicalizza la critica al vó $\mu$ os di un certo filone della prima ${ }^{37}$, recando in più un nuovo approccio all'analisi dei fenomeni della realtà umana e politica. E, beninteso, tale filosofia politica è come al solito strettamente e inestricabilmente unita ai fondamentali interessi e principi etici di Diogene.

Oltre allo specifico contenuto teorico di questa tesi, va precisato che tutto il passo presenta anche per altri aspetti un innegabile contenuto cinico: si notino il riferimento a Mida, personaggio antistenico e cinico ${ }^{38}$, la presenza del tema dell'inutilità delle ricchezze ${ }^{39}$, nonché l'opposizione tra ciò che è per natura e ciò che è per legge ${ }^{40}$; si noti infine l'ironia del ragionamento svolto a proposito del morire di fame pur possedendo ori $^{41}$.

Si potrebbe, per conferire il maggior rigore possibile alla dimostrazione che stiamo svolgendo, avanzare la supposizione che Aristotele ci renda qui testimonianza di una concezione di Antistene, la quale costituirebbe il precedente teorico delle dottrine di Diogene stesso. Ma questa ipotesi va scartata, perché da nessuna testimonianza risulta che il Socratico sia andato così avanti nella critica della polis esistente da mettere in questione una delle sue costitutive istituzioni, quella della moneta. La critica di Antistene va in tutt'altra direzione, investendo piuttosto la struttura democratica della polis, il suo confuso assemblearismo, l'assegnazione delle cariche per sorteggio ${ }^{42}$. Inoltre, nel passo in esame, è importante notare il gioco di parole istituito tra vó $\mu$ o e e vó $\mu$ เ $\mu a$. È noto

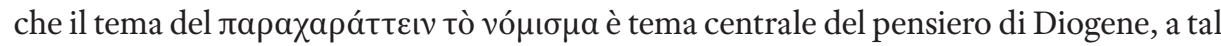
punto che lo si è potuto considerare il concetto portante di tutta la sua filosofia (Gian-

\footnotetext{
${ }^{37}$ Su questo punto fondamentale è la trattazione di Isnardi Parente (1983). Della stessa autrice si veda anche l'articolo Egualitarismo democratico nella sofistica? (Isnardi Parente 1975).

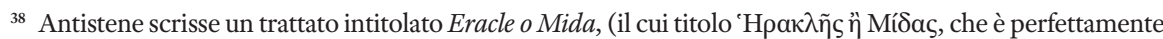

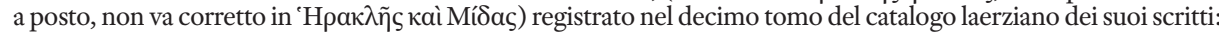
cfr. D.L. VI 18 (= SSR V A 41). Per il riferimento a Mida di Diogene cfr. Gnom. Vat. 742 n. 181 (= SSR V A 300)

39 Tema notoriamente cinico (cfr. almeno i testi SSR V B 220-246), e già antistenico: cfr. Stob. III 10, 41 (= SSR V A 80); X. Smp. 3, 8 (= SSR V A 81); ivi, 4, 34-44 (= SSR V A 82); ivi, 2, 2-5 (= SSR V A 83).

${ }^{40}$ Cfr. per Antistene, Phld. De pietate 7 ${ }^{\mathrm{a}}$, 3-8 (= SSR V A 179), e, su di esso, Brancacci (1985-1986); per Diogene cfr. soprattutto D.L. VI 71 e VI 38 (= SSR V B 7).

${ }^{41}$ Cfr. X. Smp. 4, 36 (= SSR V A 82).

${ }^{42}$ Per questo aspetto del pensiero di Antistene, mi permetto di rinviare a Brancacci (2005: 135-137), e ai miei due articoli (Brancacci 2011;2013).
} 
nantoni 1990: 513-527). Ora un testo, nel quale si fa riferimento a una posizione filosofica la quale devalorizza e svuota di significato l'istituzione della moneta, facendo leva sull'appartenenza della moneta alla sfera di vó $\mu$ os, per nulla corrispondente a quella

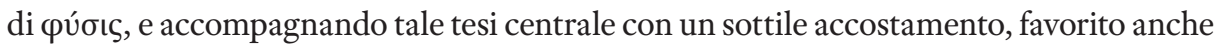

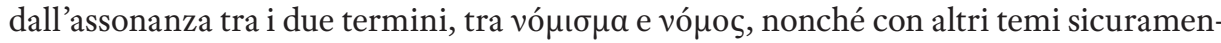
te cinici, quali il riferimento a Mida, e all'inutilità delle ricchezze, non può riferirsi che a Diogene cinico.

Non è necessario pensare che in questo passo Aristotele attinga proprio alla Repubblica di Diogene: la tesi sulla moneta qui ricordata, essendo una tesi caratteristica del

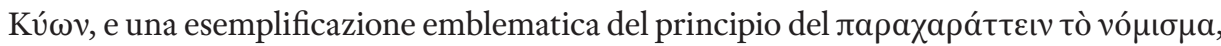
anzi forse la sua prima e fondamentale applicazione, proprio perché relativa al vó $\mu \iota \sigma \mu \alpha$, poteva essere espressa in qualunque delle sue opere, che, come mostra la testimonianza della Retorica, Aristotele è in grado di citare. Anche il fatto che Aristotele non menzioni Diogene nel noto passo del secondo libro della Politica in cui asserisce che, eccetto Platone, nessun altro filosofo ha sostenuto la comunanza delle donne ${ }^{43}$, conduce alla stessa conclusione, ovvero che la redazione della Repubblica di Diogene si situa in un periodo posteriore a quello in cui Aristotele scrive questo trattato. Ma allora, sia il passo sulla moneta preso in esame, sia gli altri del primo libro cui abbiamo fatto riferimento, suggeriscono che il progetto diogenico, improntato all'aủ á to della naturalità della polis storicamente costituita, dall'altro, e, ancora, alla messa in discussione dei rapporti padronali e matrimoniali, che sono alla base della polis stessa, e infine, e soprattutto, all'abolizione del conio corrente, trovi riscontro in un contesto storico-teorico preciso e documentabile, anteriore a Diogene stesso. Ad esso il Cinico reagirebbe polemicamente con una colossale applicazione del principio fondamentale

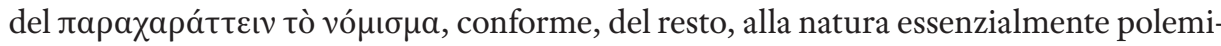
ca della sua Politeia. Per quanto attiene ai temi segnalati, è lecito pensare, dunque, che quest'ultima rappresenti anche una decostruzione di alcune tesi fondamentali sostenute in quel trattato sull'organizzazione della famiglia e sui principi fondamentali dell'economia che è il primo libro della Politica di Aristotele.

43 Arist. Pol. 1266a34-35: «nessun altro [all'infuori di Platone] ha introdotto innovazioni come la comunanza dei figli e delle donne e neppure i sissizi per le donne». 


\section{BIBLIOGRAFIA}

Aubenque, P., 1963, Le Problème de l'être chez Aristote, Paris.

BARNS, J., 1951, “A New Gnomologium”, Classical Quarterly 45, pp. 1-19.

BAroncelli, F., LAPInI, W., 2001, "Ipparchia di Maronea, sorella di Metrocle cinico, sposa di Cratete tebano, e il perfido Teodoro", Maia 53, pp. 635-642.

Bartalucci, A., 1970-1971, “Una probabile ricostruzione dell'Eracle di Diogene di Sinope”, Studi Classici e Orientali 19-20, pp. 109-122.

Brancacci, A., 1985-1986, "La Théologie d'Antisthène”, Philosophia. Yearbook of the Academy of Athens 15-16, pp. 218-230.

Brancacci, A., 1993, “Erotique et théorie du plaisir chez Antisthène”, in: M.-O. Goulet-Cazé, R. Goulet (eds.), Le cynisme ancien et ses prolongements. Actes du colloque international du CNRS (Paris, 22-25 juillet 1991), Paris, pp. 35-55.

BrancacCi, A., 2005, Antisthène. Le discours propre, Paris.

BrancaccI, A., 2011, “Antistene e Socrate in una testimonianza di Filodemo (T 17 Acosta Méndez-Angeli)”, Cronache Ercolanesi 41, pp. 83-91.

BrancaccI, A., 2013, "Introduzione al pensiero politico di Antistene", in: F. De Luise, A. Stavru (eds.), Socratica III. Studies on Socrates, the Socratics, and the Ancient Socratic Literature, Sankt Augustin, pp. 29-40.

Crisculolo, U., 1970, “Cratete di Tebe e la tradizione cinica”, Maia 22, pp. 360-367.

Decleva Caizzi, F., 1966, Antisthenis fragmenta, Milano.

Decleva Caizzi, F., 1993, “L'elogio del cane. Sesto Empirico, Schizzi pirroniani 62-78”, Elenchos 14, pp. 305-330.

DiERAUER, U., 1977, Tier und Mensch im Denken der Antike. Ideengeschichtliche Studien zur Tierpsychologie, Amsterdam.

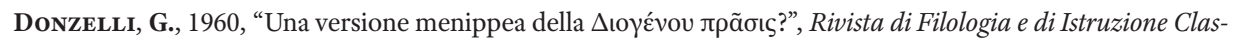
sica 88 , pp. 225-276.

Dorandi, T., 1993, “La Politeia de Diogène de Sinope et quelques remarques sur sa pensée politique”, in: M.-O. Goulet-Cazé, R. Goulet (eds.), Le cynisme ancien et ses prolongements. Actes du colloque international du CNRS (Paris, 22-25 juillet 1991), Paris, pp. 57-68.

DörING, I., 1995, “Diogenes und Antisthenes”, in: G. Giannantoni et alii (cur.), La tradizione socratica. Seminario di studi, Napoli, pp. 125-150.

DUDLEY, D. R., 1937, A History of Cynicism from Diogenes to the VI ${ }^{\text {th }}$ Century A.D., London.

DürING, I., 1966, Aristoteles, Heidelberg.

GALLO, I., 1980, Frammenti biografici da papiri, vol. II: La biografia dei filosofi, Roma.

GASTALDI, S. (cur.), 2014, Aristotele, Retorica, Roma.

Giannantoni, G., 1990, Socratis et Socraticorum reliquiae, vol. IV, Napoli.

Giannattasio ANDria, R., 1980, “Diogene cinico nei papiri ercolanesi”, Cronache Ercolanesi 10, pp. 129-151.

GoMPERz, T., 1903, Griechische Denker, Eine Geschichte der antiken Philosophie, Bd. III, Leipzig.

Goulet Cazé, M.-O., 1996, “Who was the First Dog?”, in: R. B. Branham, M.-O. Goulet Cazé (eds.), The Cynics. The Cynic Movement in Antiquity and Its Legacy, Berkeley-Los Angeles 1996, pp. 414 415.

Grote, G., 1888, Plato and the other Companions of Socrates. A New Edition, vol. III, London.

Höıstad, R., 1948, Cynic Hero and Cynic King. Studies in the Cynic Conception of Man, Lund. 
Husson, S., 2011, La République de Diogène. Une cité en quête de la nature, Paris.

ISNARdi Parente, M., 1975, “Egualitarismo democratico nella sofistica?", Rivista critica di storia della filosofia 30, 1975, pp. 3-26.

ISNardi Parente, M., 1983, "Il pensiero politico greco dalle origini alla sofistica", in: L. Firpo (dir.), Storia delle idee politiche economiche e sociali, Vol. I: L'antichità classica, Torino, pp. 127-223.

Izzo, D., 2014, “Krates bei Plautus? Persa, 118-28”, Annali Online di Ferrara 9, pp. 76-99.

LaPINI, W., 2003, Studi di filologia filosofica greca, Firenze.

Leo, F., 1906, “Diogenes bei Plautus”, Hermes 41, pp. 441-446 [anche in: F. Leo, Ausgewählte Kleine Schriften, E. Fränkel (hrsg.), Bd. I, Roma, pp. 185-190].

López CruCes, J. L., 2003, "Diógenes y sus tragedias a la luz de la comedia", Itaca. Quadernes Catalans de Cultura Clàssica 19, pp. 47-69.

LóPez CruCeS, J. L., 2010, “Une tragédie perdue; l'Héraclès de Diogène le Cynique”, Les Etudes Classiques 78 , pp. 3-24.

Moles, J., 1995, "The Cynics and politics", in: A. Laks, M. Schofield (eds), Justice and Generosity. Studies in Hellenistic Social and Political Philosophy. Proceedings of the Sixth Symposium Hellenisticum, Cambridge, pp. 129-158.

Paradiso, A., 2014, "Ipparchia cinica: la trasgressione come sillogismo", in: U. Bultrighini, E. Dimauro (cur.), Donne che contano nella storia greca, Lanciano, pp. 839-864.

Pasetri, L., 2011, "Intellettuali nel Persa? Il parassita, sua figlia e la 'filosofia da commedia”, in: R. Raffaelli, A. Tontini (cur.), Lecturae Plautinae Sarsinates XIV. Persa, Urbino, pp. 69-92.

Prince, S., 2015, Antisthenes of Athens. Text, Translations, and Commentary, Ann Arbor.

Roca Ferrer, J., 1974, “Kynikòs Tropos y los géneros literarios del helenismo”, Boletín del Instituto de Estudios Helénicos 8, pp. 163-193.

SAYRE, F., 1938, Diogenes of Sinope. A Study of Greek Cynicism, Baltimore.

Schofield, M., 1991, The Stoic Idea of the City, Cambridge.

von Fritz, K., 1926, Quellen-Untersuchungen zu Leben und Philosophie des Diogenes von Sinope, Leipzig.

von Wilamowitz-MoellendorfF, U., 1893, De tragicorum fragmentis commentatio, Göttingen [anche in: U. Von Wilamowitz-Moellendorff, Kleine Schriften, Bd. I, Berlin 1935, pp. 176-208].

WeBer, E., 1887, “De Dione Crysostomo Cynicorum sectatore”, Leipziger Studien für classischen Philologie 10, pp. $77-268$.

ZaCCaRIa, P., 2017, “The First Dog: Diogenes (Aristot. Rhet. 3,10,1411a24-25)”, Hermes 145, pp. 364-370. 
ALDO BRANCACCI

/ University of Rome Tor Vergata, Italy / aldobrancacci@yahoo.it

K E Y WOR DS

\section{Aristotle and Diogenes the Cynic}

In this paper I examine the testimonium of Aristotle's Rhetoric concerning Diogenes the Cynic (SSR V B 184). This piece of evidence is the most ancient source of Diogenes and proves that Aristotle was familiar with his writings. I also study the testimonium on Diogenes that is handed down by Theophrastus ( SSR V B 172), which confirms the interest of the ancient Peripatos in this philosopher. Finally, I examine a passage of Book 1 of the Politics where Aristotle refers to the thesis on the abolition of money. I argue that such a thesis could be ascribed to Diogenes. In particular, I attempt to demonstrate that several theses of political philosophy put forward by Diogenes should be considered as constituting a polemical overthrow of the corresponding theses of Aristotle in Book 1 of his Politics.

Aristotle, Diogenes the Cynic. Aristotles's Politics, Diogenes' Politeia, nomisma. 